\title{
Millennium Development Goals: regional perspectives - India
}

\author{
Ranjan Kumar Pejaver
}

Correspondence to Dr Ranjan Kumar Pejaver, Neonatal Medicine, KIMS and Meenakshi Hospitals, Bangalore, Karnataka, India; rpejaver@yahoo.com

Received 29 April 2014 Revised 16 October 2014 Accepted 17 October 2014
CrossMark

To cite: Pejaver RK. Arch Dis Child 2015;100

(Suppl 1):s59-s60.
India is a vast country of over one billion people. It is multilingual, multiethnic and multicultural. With advances in higher education, information technology and the knowledge industry, India has aspirations to be a developed nation by the year 2020 . This will be possible, however, only if economic progress is coupled with significant improvements in the field of healthcare. As figures $1-3$ illustrate, ${ }^{1}$ the performance of India in reaching the Millennium Development Goals (MDGs) is best described as mixed: 'moderately on track' for MDG 4 but unequivocally 'slow or off track' for MDG 5.

There are a number of complex inter-related reasons for the slower progress in India.

\section{REALITIES AND DISPARITIES}

There is a wide variation in the neonatal mortality rate (NMR) between states ranging from 11/1000 live births in Kerala to around 60/1000 in Madhya Pradesh, Uttar Pradesh, Orissa and Bihar. It is noteworthy that, in states with high levels of literacyparticularly those with empowerment of women-the NMR rates are low. There is also marked rich/poor and rural/urban discrepancy. ${ }^{2} \mathrm{NMR}$ in the poorest $20 \%$ of the population is three times that of the richest 20\% (20/1000 vs 60/1000 live births). In rural areas the NMR is more than 1.5 times that in urban areas. In rural areas $80 \%$ of the deliveries take place at home and less than half of all deliveries are attended by skilled birth attendants. However, neonatal health among the urban poor slum communities is emerging as an important problem, given the recent urban migration. Teenage marriage with its attendant problems (higher neonatal and maternal mortality) has compounded these issues, as has the controversial issue of female feticide and infanticide.

The under-five mortality rate is targeted at $42 / 1000$ live births, but India may manage to reduce it to $50 / 1000$ by $2015 .{ }^{1}$ Neonatal mortality is the major contributing factor to this. Acute respiratory tract infection and gastroenteritis are still problems in rural areas and urban slums. Improvements in infrastructure and living standards have to be achieved. Malnourishment continues to be a major hurdle. The proportion of underweight (severe and moderate) children below 3 years of age shows that it is expected to come down only to about $33 \%$ by 2015 compared with the target value of $26 \%{ }^{1}$ The percentage of the population aged between 15 and 24 years with a comprehensive correct knowledge of HIV/AIDS was $32.9 \%$ at the last survey done by National AIDS Control Organization in 2006. ${ }^{1}$

India's diversity in language, tradition, culture and practices is complex. Providing health education at the community level is a very difficult task and hinders the delivery of healthcare to its population.
Literacy is still low, especially in the villages and peripheral areas where the health indices are poor. The infrastructure such as roads, drinking water, power and communications are inadequate. There is a shortage of medicines, vaccines and ambulance, medical and nursing personnel. The money allotted to the healthcare sector is grossly inadequate.

The healthcare expenditure budget is a dismal $2 \%$ of its gross domestic product. Vast amounts of money are spent on military and security agencies to combat the external and internal insurgencies.

Although there are several federal and state government level programmes aimed towards improving perinatal health indices, effective implementation is not happening. Corruption and lack of motivation hamper progress and, perhaps unsurprisingly, in many instances only $10 \%$ of the sanctioned project budgets ultimately reaches the needy.

A strong gender bias exists at all levels with regard to utilisation of healthcare facilities; this is evident from looking at the hospital admission patterns, wages and civic representation. Women's empowerment has not been achieved.

\section{HEALTHCARE SYSTEM}

The healthcare system in India is a mix of public and private sectors. The non-governmental organisations sector plays an important but small role.

\section{Public healthcare system}

The public healthcare system consists of subcentres, primary health centres (PHCs), community health centres (CHCs), first referral units (FRUs) and district hospitals. Facility surveys show that many of the PHCs, CHCs and FRUs are not optimally functioning and are unable to provide good care to mothers, neonates and children as mandated by the programme. Traditional birth attendants are widely accepted in the communities but are limited in terms of training. Anganwadi workers belonging to the integrated child development services have a nutrition and non-formal education remit. Their role has great potential, but poor pay has led to job insecurity in this tier.

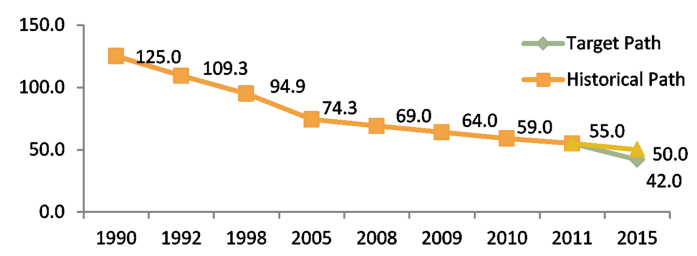

Figure 1 Under-five mortality rate (per 1000). Source: National Family Health Survey (Ministry of Health and Family Welfare), SRS (Office of Registrar General of India). 


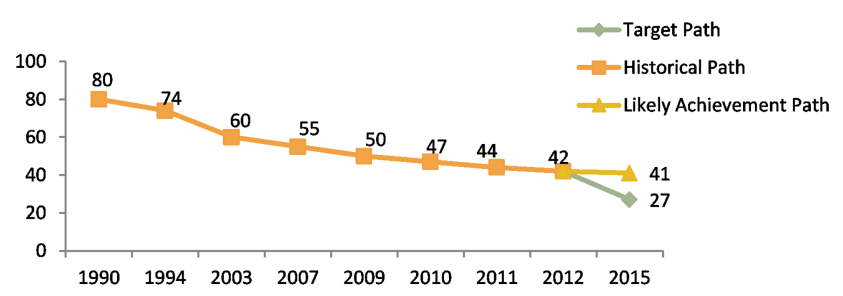

Figure 2 Infant mortality rate (per 1000 live births). Source: Office of Registrar General of India.

\section{Private healthcare sector}

India has a vast vibrant state-of-the-art private medical sector which employs $80 \%$ of the doctors. However, as they are mostly in towns and cities, they are both geographically and financially inaccessible to the majority of the population, especially as insurance is not yet sufficiently developed.

\section{WAYS FORWARD}

The two major underlying aspects which need to be carefully looked at are the literacy rate and poverty. According to the population census of 2011 , the overall literacy rate is $74.04 \%$. However, there are disparities between men (82.14\%) and women (65.46\%) and between urban areas (84.98\%) and rural areas $(68.91 \%)$ which need to be bridged. ${ }^{3}$

According to the poverty estimates of 2011-2012 in a Report of the Planning Commission, the poverty head count ratio is $21.9 \%$, so India has already achieved the target of $23.9 \%$. However, the depth of poverty as reflected by the poverty gap ratio is 5.05 for rural areas and 2.70 for urban areas of India. Even if taken separately, the gap between the rich and poor in both areas is very wide.

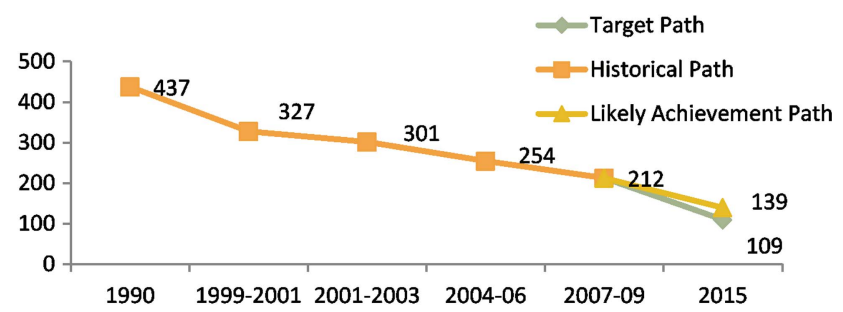

Figure 3 Maternal mortality ratio (per 100000 live births). Source: Office of Registrar General of India.
The tasks ahead are as follows:

- To improve social awareness and provide health education, especially at the community level: women's empowerment should be given high priority and gender equality should be promoted.

- To strengthen the existing programmes and policies: advocacy is essential and the political establishment must be convinced of the problems and motivated to take suitable action with a commensurate increase in the budget.

- To develop sustainable models of obstetric, neonatal and child health services: institutional deliveries to be encouraged and scale-up of community skilled birth attendants.

- To upgrade the CHC and FRUs: vacancies for doctors, nurses and paramedical workers must be filled.

- To promote breast feeding practices and strengthen babyfriendly hospital initiatives and kangaroo mother care: costeffective methods to improve child survival should be prioritised.

- To institute an action plan to address the unfinished agenda of elimination of malnutrition and communicable diseases: programmes such as the Integrated Management of Neonatal and Childhood Illnesses (IMNCI) have to be strengthened.

- To strengthen the capacity for maternal, child and newborn nursing care: structured focused training (cadre of nurse practioners) could to some extent alleviate the lack of doctors in the periphery.

- To promote better dialogue between the Government, private/corporate sectors and non-governmental organisations to avoid duplication of programmes and better utilisation of resources.

- To ensure that insurance is affordable through government or private programmes.

A lot is being done, but much more needs to be done if India's perinatal indices are to improve.

Competing interests None.

Provenance and peer review Commissioned; internally peer reviewed.

\section{REFERENCES}

1 Towards achieving Millennium Development Goals. India, 2013. Ministry of Statistics and Programme Implementation, Government of India.

2 National Family Health Survey (NFHS). District Level Household and Facility Survey (DLHS). 2010. Ministry of Health and Family Welfare, Government of India.

3 Report of the Registrar General of India. 2012. 